\title{
Eine Entscheidungshilfe zum Aussschluss einer Pneumonie reduziert unnötige Antibiotika
}

\author{
Markun, Stefan
}

DOI: https://doi.org/10.1024/1661-8157/a001473

Posted at the Zurich Open Repository and Archive, University of Zurich ZORA URL: https://doi.org/10.5167/uzh-90674

Journal Article

Accepted Version

Originally published at:

Markun, Stefan (2013). Eine Entscheidungshilfe zum Aussschluss einer Pneumonie reduziert unnötige Antibiotika. Praxis, 102(23):1430-1431.

DOI: https://doi.org/10.1024/1661-8157/a001473 


\title{
Eine Entscheidungshilfe zum Ausschluss einer Pneumonie reduziert unnötige Antibiotika
}

\author{
Frage: \\ Lässt sich für Patienten mit Infektsymptomen der Atemwege eine Entscheidungsregel \\ formulieren, die unnötige Antibiotikaverschreibungen reduziert?

\section{Hintergrund:} \\ Infektionen der Atemwege sind äusserst häufige Konsultationsgründe. Die Fälle zu ermitteln, in \\ denen eine Pneumonie vorliegt mit Bedarf einer antibiotischen Behandlung ist eine \\ verantwortungsvolle Aufgabe. Eine eher zu grosszügige Verschreibungspraxis von Antibiotika \\ wird immer wieder moniert. Antibiotika grosszügig zu verschreiben reduziert zwar die \\ Wahrscheinlichkeit beim Individuum eine potentiell schwere Krankheit unbehandelt zu lassen, \\ gleichzeitig trägt diese Praxis aber zum wachsenden Problem der Antibiotikaresistenzen bei. \\ Das Ziel dieser Studie ist es eine Entscheidungsregel zu entwickeln, die basierend auf \\ klinischen Befunden und CRP eine Pneumonie ausschliessen kann und somit helfen kann \\ unnötige Antibiotikaverschreibungen zu reduzieren.
}

\section{Einschlusskriterien:}

- Mindestens 18 Jahre alte Patienten

- Seit mindestens 24 Stunden neuer Husten oder verschlimmerter Husten

- Subjektiv oder gemessen erhöhte Körpertemperatur

\section{Ausschlusskriterien:}

- Patienten mit chronischen Lungenkrankheiten ausser chronischer Bronchitis

- Patienten, die während eines Spitalaufenthaltes Husten und Fieber entwickelten

- HIV positive Patienten, Einnahme von oralen Steroiden innerhalb des vergangenen Monats, laufende Chemotherapie, St.n. Organtransplantation

\section{Studiendesign und Methode:}

Prospektive Kohortenstudie; Statistische Ableitung einer klinisch praktikablen Entscheidungsregel basierend auf den erhobenen Daten in der Studie; Vergleich zwischen tatsächlicher Verschreibungsrate von Antibiotika mit der Verschreibungsrate unter hypothetischer Anwendung der Entscheidungsregel.

\section{Studienort:}

Hausarztpraxen und Spitäler in der Ostschweiz

\section{Interventionen:}

- Bei allen Patienten wurde die klinische Abklärung und Therapieentscheidung unabhängig von der Studie durchgeführt. Für die Studie wurden jedoch in jedem Fall zusätzlich die klinischen Befunde standardisiert dokumentiert, sowie CRP abgenommen und ein ThoraxRöntgen durchgeführt. Die Röntgenbilder wurden von Radiologen befundet, die gegenüber den klinischen Informationen verblindeten waren. Als tatsächliche Pneumonien wurden Fälle definiert mit Symptomen einer Infektion der unteren Atemwege und radiologischem Infiltrat ohne plausible andere Erklärung.

\section{Outcome:}

- Prozentsatz der vermeidbaren Antibiotikaverschreibungen

Resultat: 
- 86 Ärzte nahmen an der Studie teil, 642 Patienten (50\% Frauen, mittleres Alter 47 Jahre) konnten eingeschlossen werden, 96\% der Patienten wurden in Hausarztpraxen rekrutiert, $4 \%$ in der Notfallstation eines Spitals.

- Im Mittel hatten die Patienten seit 7 Tagen Husten, alle Patienten empfanden subjektiv eine erhöhte Körpertemperatur, die mittlere Körpertemperatur war $37.4^{\circ}, 14 \%$ der Patienten hatten bei der Konsultation Temperatur $38.5^{\circ}$ oder mehr. Radiologische Zeichen einer Pneumonie fanden sich bei $20.5 \%$ der Patienten. Bei $58 \%$ aller Patienten wurden Antibiotika verschrieben. Die Verschreibungsrate von Antibiotika bei Patienten ohne radiologische Zeichen einer Pneumonie war 38\%.

- Mit statistischen Methoden wurde aus den Daten folgende Regel zum Ausschluss einer Pneumonie abgeleitet: Eine Pneumonie kann mit grosser Sicherheit ausgeschlossen werden bei CRP $<10 \mu \mathrm{g} / \mathrm{ml}$, oder bei CRP $11-50 \mu \mathrm{g} / \mathrm{ml}$ bei gleichzeitiger Abwesenheit von Dyspnoe und täglichem Fieber.

- Die Anwendung dieser Entscheidungsregel hätte bei den Patienten in dieser Studie die Antibiotikaverschreibungen um $9.1 \%$ reduzieren können.

\section{Kommentar:}

- Die Studie berechnet ein klinisch einfach handhabbares diagnostisches Instrument, mit welchem sich basierend auf klinischen Befunden und CRP, ohne Röntgen-Thorax eine Pneumonie ausschliessen lässt. Somit liessen sich nicht nur fast $10 \%$ der Antibiotikaverschreibungen einsparen, sondern auch Röntgenuntersuchungen.

- Limitierend für alle Studien mit ähnlichem Design, ist dass Sie die Entscheidungsregel im selben Patientenset geprüft wurde, in dem sie entwickelt wurde. Es besteht also die Gefahr eines Zirkelschlusses, die Übertragbarkeit der Entscheidungsregel auf Patienten ausserhalb der Studie ist somit nicht ohne weiteres gegeben. In einer konsekutiven Validierungsstudie mit 110 neuen Patienten aus Hausarztpraxen erwies sich die Entscheidungsregel als sehr zuverlässig mit einer Rate von verpassten Pneumonien von nur 1.8\%.

- Das Problem der zunehmenden Antibiotikaresistenzen ist bekannt und unbestritten. In Spitälern sind Zusammenhänge zwischen Verschreibungspraxis und Resistenzentwicklung besonders einfach aufzuzeigen, eine Beweiskette, die den Spitälern in der öffentlichen Wahrnehmung bislang die hauptsächliche Verantwortlichkeit für das Problem einbrachte. Der quantitative Beitrag zum Problem der unterschiedlichen Instanzen, die Antibiotika abgeben ist jedoch unbekannt. Die Tierhaltung kommt durch mikrobiologische Studien zunehmend unter Druck, der Beitrag zur Resistenzentwicklung aus Hausarztpraxis ist kaum untersucht.

- Für einen verhältnismässigen Einsatz von Antibiotika sind alle verschreibenden Instanzen verantwortlich. Initiativen zur Reduktion unnötiger Verschreibungen sind vor dem Hintergrund wachsender Resistenzen aus Sicht der öffentlichen Gesundheit sehr willkommen. Im Einzelfall erübrigt sich diese Sicht aber insbesondere dann, wenn Antibiotika a priori nicht nötig sind. Diese in solchen Situationen einzusetzen ist aufgrund der potentiellen Nebenwirkungen an sich schon schwer zu rechtfertigen.

\section{Literatur:}

Steurer J, Held U, Spaar A, et al. A decision aid to rule out pneumonia and reduce unnecessary prescriptions of antibiotics in primary care patients with cough and fever. BMC Med. 2011;9:56

Verfasser:

Stefan Markun 